Proyecciones Journal of Mathematics

Vol. 30, No 2, pp. 189-199, August 2011.

Universidad Católica del Norte

Antofagasta - Chile

\title{
Difference sequence spaces defined by a sequence of modulus functions
}

\author{
KULDIP RAJ \\ SHRI MATA VAISHNO DEVI UNIVERSITY, INDIA \\ and \\ SUNIL K. SHARMA \\ SHRI MATA VAISHNO DEVI UNIVERSITY, INDIA \\ Received: March 2011. Accepted : May 2011
}

\begin{abstract}
In the present paper we study difference sequence spaces defined by a sequence of modulus functions and examine some topological properties of these spaces.
\end{abstract}

Subjclass[2000] :40A05, 40C05, 46A45.

Keywords : Paranorm space, Difference sequence space, Modulus function. 


\section{Introduction and Preliminaries}

A modulus function is a function $f:[0, \infty) \rightarrow[0, \infty)$ such that

1. $f(x)=0$ if and only if $x=0$,

2. $f(x+y) \leq f(x)+f(y)$ for all $x \geq 0, y \geq 0$,

3. $f$ is increasing,

4. $f$ is continuous from right at 0 .

It follows that $f$ must be continuous everywhere on $[0, \infty)$. The modulus function may be bounded or unbounded. For example, if we take $f(x)=\frac{x}{x+1}$, then $f(x)$ is bounded. If $f(x)=x^{p}, 0<p<1$, then the modulus $f(x)$ is unbounded. Subsequentially, modulus function has been discussed in ([1], [7], [8]) and many others.

Let $X$ be a linear metric space. A function $p: X \rightarrow \mathbf{R}$ is called paranorm, if

1. $p(x) \geq 0$, for all $x \in X$,

2. $p(-x)=p(x)$, for all $x \in X$,

3. $p(x+y) \leq p(x)+p(y)$, for all $x, y \in X$,

4. if $\left(\lambda_{n}\right)$ is a sequence of scalars with $\lambda_{n} \rightarrow \lambda$ as $n \rightarrow \infty$ and $\left(x_{n}\right)$ is a sequence of vectors with $p\left(x_{n}-x\right) \rightarrow 0$ as $n \rightarrow \infty$, then $p\left(\lambda_{n} x_{n}-\right.$ $\lambda x) \rightarrow 0$ as $n \rightarrow \infty$.

A paranorm $p$ for which $p(x)=0$ implies $x=0$ is called total paranorm and the pair $(X, p)$ is called a total paranormed space. It is well known that the metric of any linear metric space is given by some total paranorm (see [9], Theorem 10.4.2, P-183).

Let $w$ be the set of all sequences, real or complex numbers and $l_{\infty}, c$ and $c_{0}$ be respectively the Banach spaces of bounded, convergent and null sequences $x=\left(x_{k}\right)$, normed by $\|x\|=\sup _{k}\left|x_{k}\right|$, where $k \in \mathbf{N}$, the set of positive integers. 
Let $\Lambda=\left(\lambda_{n}\right)$ be a non decreasing sequence of positive reals tending to infinity and $\lambda_{1}=1$ and $\lambda_{n+1} \leq \lambda_{n}+1$. The generalized de la Vallee-Poussin means is defined by

$$
t_{n}(x)=\frac{1}{\lambda_{n}} \sum_{k \in I_{n}} x_{k}
$$

where $I_{n}=\left[n-\lambda_{n}+1, n\right]$. A sequence $x=\left(x_{k}\right)$ is said to be $(V, \lambda)$-summable to a number $l$ if $t_{n}(x) \rightarrow l$ as $n \rightarrow \infty$ (see[5]). If $\lambda_{n}=n,(V, \lambda)$-summability and strong $(V, \lambda)$-summability are reduced to $(C, 1)$-summability and $[C, 1]$ summability, respectively.

In [4], Kizmaz defined the sequence spaces

$$
X(\Delta)=\left\{x=\left(x_{k}\right):\left(\Delta x_{k}\right) \in X\right\}
$$

for $X=l_{\infty}, c$ or $c_{0}$, where $\Delta x=\left(\Delta x_{k}\right)=\left(x_{k}-x_{k+1}\right)$ for all $k \in \mathbf{N}$.

Et and Colak [2] generalized the above sequence spaces to the sequence spaces

$$
X\left(\Delta^{m}\right)=\left\{x=\left(x_{k}\right):\left(\Delta^{m} x_{k}\right) \in X\right\}
$$

for $X=l_{\infty}, c$ or $c_{0}$, where $m \in \mathbf{N}, \Delta^{0} x=\left(x_{k}\right), \Delta x=\left(x_{k}-x_{k+1}\right)$,

$$
\Delta^{m} x=\left(\Delta^{m} x_{k}\right)=\left(\Delta^{m-1} x_{k}-\Delta^{m-1} x_{k+1}\right) \quad \text { for all } k \in \mathbf{N} \text {. }
$$

The generalized difference operator has the following binomial representation,

$$
\Delta^{m} x_{k}=\sum_{v=0}^{m}(-1)^{v}\left(\begin{array}{c}
m \\
v
\end{array}\right) x_{k+v}
$$

for all $k \in \mathbf{N}$.

The following inequality will be used throughout the paper. If $0 \leq p_{k} \leq$ $\sup p_{k}=H, D=\max \left(1,2^{H-1}\right)$ then

$$
\left|a_{k}+b_{k}\right|^{p_{k}} \leq D\left\{\left|a_{k}\right|^{p_{k}}+\left|b_{k}\right|^{p_{k}}\right\}
$$

for all $k$ and $a_{k}, b_{k} \in \mathbf{C}$. Also $|a|^{p_{k}} \leq \max \left(1,|a|^{H}\right)$ for all $a \in \mathbf{C}$. 
Let $E$ be a Banach space, we define $w(E)$ to be the vector space of all $E$-valued sequences that is

$$
w(E)=\left\{x=\left(x_{k}\right): x_{k} \in E\right\}
$$

Let $F=\left(f_{k}\right)$ be a sequence of modulus functions and $p=\left(p_{k}\right)$ be a bounded sequence of positive real numbers. Then we define the following sequence spaces :

$$
\begin{gathered}
{[V, \lambda, F, p]_{1}\left(\Delta^{m}, E, u\right)=\left\{x \in w(E): \lim _{n} \frac{1}{\lambda_{n}} \sum_{k \in I_{n}}\left[f_{k}\left(\left\|\Delta^{m} u_{k} x_{k}-L e\right\|\right)\right]^{p_{k}}\right.} \\
=0, \text { for some } L\}, \\
{[V, \lambda, F, p]_{0}\left(\Delta^{m}, E, u\right)=\left\{x \in w(E): \lim _{n} \frac{1}{\lambda_{n}} \sum_{k \in I_{n}}\left[f_{k}\left(\left\|\Delta^{m} u_{k} x_{k}\right\|\right)\right]^{p_{k}}=0\right\}}
\end{gathered}
$$

and

$[V, \lambda, F, p]_{\infty}\left(\Delta^{m}, E, u\right)=\left\{x \in w(E): \sup _{n} \frac{1}{\lambda_{n}} \sum_{k \in I_{n}}\left[f_{k}\left(\left\|\Delta^{m} u_{k} x_{k}\right\|\right)\right]^{p_{k}}<\infty\right\}$,

where $e=(1,1,1, \cdots)$.

If $u=e$ and $f_{k}=f$, then these spaces reduce to those which were studied by Et, M., Altin, Y. and Altinok, H. [3].

For $f_{k}(x)=x$, we have

$$
\begin{gathered}
{[V, \lambda, p]_{1}\left(\Delta^{m}, E, u\right)=\left\{x \in w(E): \lim _{n} \frac{1}{\lambda_{n}} \sum_{k \in I_{n}}\left[\left\|\Delta^{m} u_{k} x_{k}-L e\right\|\right]^{p_{k}}=0\right.} \\
\text { for some } L\} \\
{[V, \lambda, p]_{0}\left(\Delta^{m}, E, u\right)=\left\{x \in w(E): \lim _{n} \frac{1}{\lambda_{n}} \sum_{k \in I_{n}}\left[\left\|\Delta^{m} u_{k} x_{k}\right\|\right]^{p_{k}}=0\right\}}
\end{gathered}
$$

and

$$
[V, \lambda, p]_{\infty}\left(\Delta^{m}, E, u\right)=\left\{x \in w(E): \sup _{n} \frac{1}{\lambda_{n}} \sum_{k \in I_{n}}\left[\left\|\Delta^{m} u_{k} x_{k}\right\|\right]^{p_{k}}<\infty\right\} .
$$


For $p_{k}=1$, we have

$$
\begin{aligned}
& {[V, \lambda, F]_{1}\left(\Delta^{m}, E, u\right)=\left\{x \in w(E): \lim _{n} \frac{1}{\lambda_{n}} \sum_{k \in I_{n}}\left[f_{k}\left(\left\|\Delta^{m} u_{k} x_{k}-L e\right\|\right)\right]=0\right.} \\
& \text { for some } L\} \\
& {[V, \lambda, F]_{0}\left(\Delta^{m}, E, u\right)=\left\{x \in w(E): \lim _{n} \frac{1}{\lambda_{n}} \sum_{k \in I_{n}}\left[f_{k}\left(\left\|\Delta^{m} u_{k} x_{k}\right\|\right)\right]=0\right\}}
\end{aligned}
$$

and

$$
[V, \lambda, F]_{\infty}\left(\Delta^{m}, E, u\right)=\left\{x \in w(E): \sup _{n} \frac{1}{\lambda_{n}} \sum_{k \in I_{n}}\left[f_{k}\left(\left\|\Delta^{m} u_{k} x_{k}\right\|\right)\right]<\infty\right\} .
$$

For $f_{k}(x)=x$ and $p_{k}=1$ for all $k \in \mathbf{N}$, we have

$$
\begin{gathered}
{[V, \lambda]_{1}\left(\Delta^{m}, E, u\right)=\left\{x \in w(E): \lim _{n} \frac{1}{\lambda_{n}} \sum_{k \in I_{n}}\left[\left\|\Delta^{m} u_{k} x_{k}-L e\right\|\right]=0,\right.} \\
\quad \text { for some } L\} \\
{[V, \lambda]_{0}\left(\Delta^{m}, E, u\right)=\left\{x \in w(E): \lim _{n} \frac{1}{\lambda_{n}} \sum_{k \in I_{n}}\left[\left\|\Delta^{m} u_{k} x_{k}\right\|\right]=0\right\}}
\end{gathered}
$$

and

$$
[V, \lambda]_{\infty}\left(\Delta^{m}, E, u\right)=\left\{x \in w(E): \sup _{n} \frac{1}{\lambda_{n}} \sum_{k \in I_{n}}\left[\left\|\Delta^{m} u_{k} x_{k}\right\|\right]<\infty\right\} .
$$

Throughout this paper, $X$ will denote any one of the notations 0,1 or $\infty$.

In this paper we study some topological properties and inclusion relations between above defined sequence spaces.

\section{Main Results}

Theorem 2.1 Let $F=\left(f_{k}\right)$ be a sequence of modulus functions and $p=$ $\left(p_{k}\right)$ be a bounded sequence of positive real numbers. Then the sequence spaces $[V, \lambda, F, p]_{1}\left(\Delta^{m}, E, u\right),[V, \lambda, F, p]_{0}\left(\Delta^{m}, E, u\right)$ and $[V, \lambda, F, p]_{\infty}\left(\Delta^{m}, E, u\right)$ are linear spaces. 
Proof. Let $x, y \in[V, \lambda, F, p]_{0}\left(\Delta^{m}, E, u\right)$ and $\alpha, \beta \in \mathbf{C}$. Then there exist positive number $M_{\alpha}$ and $N_{\beta}$ such that $|\alpha| \leq M_{\alpha}$ and $|\beta| \leq N_{\beta}$. Since $f_{k}$ is subadditive and $\Delta^{m}$ is linear, we have

$$
\begin{aligned}
& \quad \frac{1}{\lambda_{n}} \sum_{k \in I_{n}}\left[f_{k}\left(\left\|\Delta^{m}\left(\alpha u_{k} x_{k}+\beta u_{k} y_{k}\right)\right\|\right)\right]^{p_{k}} \\
& \leq \frac{1}{\lambda_{n}} \sum_{k \in I_{n}}\left[f_{k}\left(|\alpha|\left\|\Delta^{m} u_{k} x_{k}\right\|\right)+f_{k}\left(|\beta| \| \Delta^{m} u_{k} y_{k}\right)\right]^{p_{k}} \\
& \leq D\left(M_{\alpha}\right)^{H} \frac{1}{\lambda_{n}} \sum_{k \in I_{n}}\left[f_{k}\left(\| \Delta^{m} u_{k} x_{k}\right)\right]^{p_{k}}+D\left(N_{\beta}\right)^{H} \frac{1}{\lambda_{n}} \sum_{k \in I_{n}}\left[f_{k}\left(\left\|\Delta^{m} u_{k} y_{k}\right\|\right)\right]^{p_{k}} \\
& \rightarrow 0 \text { as } n \rightarrow \infty .
\end{aligned}
$$

This proves that $[V, \lambda, F, p]_{0}\left(\Delta^{m}, E, u\right)$ is a linear space. Similarly we can prove that $[V, \lambda, F, p]_{1}\left(\Delta^{m}, E, u\right)$ and $[V, \lambda, F, p]_{\infty}\left(\Delta^{m}, E, u\right)$ are linear spaces in view of the above proof.

Theorem 2.2 Let $F=\left(f_{k}\right)$ be a sequence of modulus functions. Then $[V, \lambda, F, p]_{0}\left(\Delta^{m}, E, u\right) \subset[V, \lambda, F, p]_{1}\left(\Delta^{m}, E, u\right) \subset[V, \lambda, F, p]_{\infty}\left(\Delta^{m}, E, u\right)$.

Proof. The first inclusion is obvious. For the second inclusion, let $x \in[V, \lambda, F, p]_{1}\left(\Delta^{m}, E, u\right)$. Then by definition, we have

$$
\begin{aligned}
\frac{1}{\lambda_{n}} \sum_{k \in I_{n}} & {\left[f_{k}\left(\left\|\Delta^{m} u_{k} x_{k}\right\|\right)\right]^{p_{k}} } \\
& =\frac{1}{\lambda_{n}} \sum_{k \in I_{n}}\left[f_{k}\left(\| \Delta^{m} u_{k} x_{k}-L e+L e\right)\right]^{p_{k}} \\
& \leq D \frac{1}{\lambda_{n}} \sum_{k \in I_{n}}\left[f_{k}\left(\left\|\Delta^{m} u_{k} x_{k}-L e\right\|\right)\right]^{p_{k}}+D \frac{1}{\lambda_{n}} \sum_{k \in I_{n}}\left[f_{k}(\|L e\|)\right]^{p_{k}} .
\end{aligned}
$$

Now, there exists a positive number $A$ such that $\|L e\| \leq A$. Hence we have

$$
\frac{1}{\lambda_{n}} \sum_{k \in I_{n}}\left[f_{k}\left(\left\|\Delta^{m} u_{k} x_{k}\right\|\right)\right]^{p_{k}} \leq \frac{D}{\lambda_{n}} \sum_{k \in I_{n}}\left[f_{k}\left(\left\|\Delta^{m} u_{k} x_{k}-L e\right\|\right)\right]^{p_{k}}+
$$




$$
\frac{D}{\lambda_{n}}[A]^{H} \sum_{k \in I_{n}}\left[f_{k}(1)\right]^{H} .
$$

Since $x \in[V, \lambda, F, p]_{1}\left(\Delta^{m}, E, u\right)$ we have $x \in[V, \lambda, F, p]_{\infty}\left(\Delta^{m}, E, u\right)$. Therefore,

$$
[V, \lambda, F, p]_{1}\left(\Delta^{m}, E, u\right) \subset[V, \lambda, F, p]_{\infty}\left(\Delta^{m}, E, u\right) .
$$

This completes the proof.

Theorem 2.3 Let $F=\left(f_{k}\right)$ be a sequence of modulus functions and $p=$ $\left(p_{k}\right)$ be a bounded sequence of positive real numbers. Then $[V, \lambda, F, p]_{0}\left(\Delta^{m}, E, u\right)$ is a paranormed space with

$$
g_{\Delta}(x)=\sup _{n}\left(\frac{1}{\lambda_{n}} \sum_{k \in I_{n}}\left[f_{k}\left(\left\|\Delta^{m} u_{k} x_{k}\right\|\right)\right]^{p_{k}}\right)^{\frac{1}{K}}
$$

where $K=\max \left(1, \sup p_{k}\right)$.

Proof. Clearly $g_{\Delta}(x)=g_{\Delta}(-x)$. It is trivial that $\Delta^{m} u_{k} x_{k}=0$ for $x=0$. Since $f(0)=0$, we get $g_{\Delta}(x)=0$ for $x=0$. Since $\frac{p_{k}}{K} \leq 1$, using the Minkowski's inequality, for each $n$, we have

$$
\begin{aligned}
& \left(\frac{1}{\lambda_{n}} \sum_{k \in I_{n}}\left[f_{k}\left(\left\|\Delta^{m} u_{k} x_{k}+\Delta^{m} u_{k} y_{k}\right\|\right)\right]^{p_{k}}\right)^{\frac{1}{K}} \\
& \leq\left(\frac{1}{\lambda_{n}} \sum_{k \in I_{n}}\left[f_{k}\left(\| \Delta^{m} u_{k} x_{k}\right)+f_{k}\left(\left\|\Delta^{m} u_{k} y_{k}\right\|\right)\right]^{p_{k}}\right)^{\frac{1}{K}} \\
& \leq\left(\frac{1}{\lambda_{n}} \sum_{k \in I_{n}}\left[f_{k}\left(\left\|\Delta^{m} u_{k} x_{k}\right\|\right)\right]^{p_{k}}\right)^{\frac{1}{K}}+\left(\frac{1}{\lambda_{n}} \sum_{k \in I_{n}}\left[f_{k}\left(\left\|\Delta^{m} u_{k} y_{k}\right\|\right)\right]^{p_{k}}\right)^{\frac{1}{K}} .
\end{aligned}
$$

Hence $g_{\Delta}(x)$ is subadditive. For, the continuity of multiplication, let us take any complex number $\alpha$. By definition, we have

$$
\begin{aligned}
g_{\Delta}(\alpha x) & =\sup _{n}\left(\frac{1}{\lambda_{n}} \sum_{k \in I_{n}}\left[f_{k}\left(\left\|\Delta^{m} \alpha u_{k} x_{k}\right\|\right)\right]^{p_{k}}\right)^{\frac{1}{K}} \\
& \leq C_{\alpha}^{H / K} g_{\Delta}(x),
\end{aligned}
$$


where $C_{\alpha}$ is a positive integer such that $|\alpha| \leq C_{\alpha}$. Now, let $\alpha \rightarrow 0$ for any fixed $x$ with $g_{\Delta}(x) \neq 0$. By definition for $|\alpha|<1$, we have

$$
\frac{1}{\lambda_{n}} \sum_{k \in I_{n}}\left[f_{k}\left(\left\|\alpha \Delta^{m} u_{k} x_{k}\right\|\right)\right]^{p_{k}}<\epsilon \text { for } n>n_{0}(\epsilon)
$$

Also, for $1 \leq n \leq n_{0}$, taking $\alpha$ small enough, since $f_{k}$ is continuous, we have

$$
\frac{1}{\lambda_{n}} \sum_{k \in I_{n}}\left[f_{k}\left(\left\|\alpha \Delta^{m} u_{k} x_{k}\right\|\right)\right]^{p_{k}}<\epsilon .
$$

Now, eqn. (2.2) and (2.3) together imply that

$$
g_{\Delta}(\alpha x) \rightarrow 0 \text { as } \alpha \rightarrow 0
$$

Theorem 2.4 Let $F=\left(f_{k}\right)$ be a sequence of modulus functions and $m \geq 1$, then the inclusion

$$
[V, \lambda, F]_{X}\left(\Delta^{m-1}, E, u\right) \subset[V, \lambda, F]_{X}\left(\Delta^{m}, E, u\right)
$$

is strict. In general

$$
[V, \lambda, F]_{X}\left(\Delta^{i}, E, u\right) \subset[V, \lambda, F]_{X}\left(\Delta^{m}, E, u\right)
$$

for all $i=1,2, \cdots, m-1$ and the inclusion is strict.

Proof. Let $x \in[V, \lambda, F]_{\infty}\left(\Delta^{m-1}, E, u\right)$. Then we have

$$
\sup _{n} \frac{1}{\lambda_{n}} \sum_{k \in I_{n}}\left[f_{k}\left(\left\|\Delta^{m-1} u_{k} x_{k}\right\|\right)\right]<\infty \text {. }
$$

By definition, we have

$$
\begin{gathered}
\frac{1}{\lambda_{n}} \sum_{k \in I_{n}}\left[f_{k}\left(\left\|\Delta^{m} u_{k} x_{k}\right\|\right)\right]=\frac{1}{\lambda_{n}} \sum_{k \in I_{n}}\left[f_{k}\left(\left\|\Delta^{m-1} u_{k} x_{k}\right\|\right)\right]+ \\
\frac{1}{\lambda_{n}} \sum_{k \in I_{n}}\left[f_{k}\left(\left\|\Delta^{m-1} u_{k+1} x_{k+1}\right\|\right)\right] \leq \infty .
\end{gathered}
$$

Thus $[V, \lambda, F]_{\infty}\left(\Delta^{m-1}, E, u\right) \subset[V, \lambda, F]_{\infty}\left(\Delta^{m}, E, u\right)$. 
Proceeding in this way, we have

$$
[V, \lambda, F]_{\infty}\left(\Delta^{i}, E, u\right) \subset[V, \lambda, F]_{\infty}\left(\Delta^{m}, E, u\right)
$$

for all $i=1,2, \cdots, m-1$. Let $E=\mathbf{C}$ and $\lambda_{n}=n$ for each $n \in \mathbf{N}$. Then the sequence $x=\left(x^{m}\right) \in[V, \lambda, F]_{\infty}\left(\Delta^{m}, E, u\right)$ but does not belong to $[V, \lambda, F]_{\infty}\left(\Delta^{m-1}, E, u\right)$ for $f_{k}(x)=x$.

Similarly, we can prove for the case $[V, \lambda, F]_{0}\left(\Delta^{m}, E, u\right)$ and $[V, \lambda, F]_{1}\left(\Delta^{m}, E, u\right)$ in view of the above proof.

Corollary 2.5 Let $F=\left(f_{k}\right)$ be a sequence of modulus functions. Then

$$
[V, \lambda, F, p]_{1}\left(\Delta^{m-1}, E, u\right) \subset[V, \lambda, F]_{0}\left(\Delta^{m}, E, u\right) .
$$

Theorem 2.5 Let $F=\left(f_{k}\right), F^{\prime}=\left(f_{k}^{\prime}\right)$ and $F^{\prime \prime}=\left(f_{k}^{\prime \prime}\right)$ are sequence of modulus functions. Then we have

(i) $\left[V, \lambda, F^{\prime}, p\right]_{X}\left(\Delta^{m}, E, u\right) \subset\left[V, \lambda, F \circ F^{\prime}, p\right]_{X}\left(\Delta^{m}, E, u\right)$,

(ii) $\left[V, \lambda, F^{\prime}, p\right]_{X}\left(\Delta^{m}, E, u\right) \cap\left[V, \lambda, F^{\prime \prime}, p\right]_{X}\left(\Delta^{m}, E, u\right) \subset\left[V, \lambda, F+F^{\prime}, p\right]_{X}\left(\Delta^{m}, E, u\right)$.

Proof. (i) Let $\epsilon>0$ and choose $\delta$ with $0<\delta<1$ such that $f(t)<\epsilon$ for $0 \leq t \leq \delta$. Write $y_{k}=f_{k}^{\prime}\left(\left\|\Delta^{m} u_{k} x_{k}\right\|\right)$ and consider

$$
\sum_{k \in I_{n}}\left[f_{k}\left(y_{k}\right)\right]^{p_{k}}=\sum_{1}\left[f_{k}\left(y_{k}\right)\right]^{p_{k}}+\sum_{2}\left[f_{k}\left(y_{k}\right)\right]^{p_{k}},
$$

where the first summation is over $y_{k} \leq \delta$ and second summation is over $y_{k} \geq \delta$. Since $f_{k}$ is continuous, we have

$$
\sum_{1}\left[f_{k}\left(y_{k}\right)\right]^{p_{k}}<\lambda_{n} \epsilon^{H}
$$

and for $y_{k}>\delta$, we use the fact that

$$
y_{k}<\frac{y_{k}}{\delta} \leq 1+\frac{y_{k}}{\delta}
$$

By the definition, we have for $y_{k}>\delta$,

$$
f_{k}\left(y_{k}\right)<2 f_{k}(1) \frac{y_{k}}{\delta} \text {. }
$$


Hence

$$
\frac{1}{\lambda_{n}} \sum_{2}\left[f_{k}\left(y_{k}\right)\right]^{p_{k}} \leq \max \left(1,\left(2 f_{k}(1) \delta^{-1}\right)^{H}\right) \frac{1}{\lambda_{n}} \sum_{k \in I_{n}} y_{k} .
$$

From eqn. (2.4) and (2.5), we have

$$
[V, \lambda, F, p]_{0}\left(\Delta^{m}, E, u\right) \subset\left[V, \lambda, F \circ F^{\prime}, p\right]_{0}\left(\Delta^{m}, E, u\right) .
$$

This completes the proof of (i).

The proof of (ii) follows from the following inequality:

$$
\left[\left(f_{k}^{\prime}+f_{k}^{\prime \prime}\right)\left(\left\|\Delta^{m} u_{k} x_{k}\right\|\right)\right]^{p_{k}} \leq D\left[f_{k}^{\prime}\left(\left\|\Delta^{m} u_{k} x_{k}\right\|\right)\right]^{p_{k}}+D\left[f_{k}^{\prime \prime}\left(\left\|\Delta^{m} u_{k} x_{k}\right\|\right)\right]^{p_{k}} .
$$

Corollary 2.6 Let $F=\left(f_{k}\right)$ be a sequence of modulus functions. Then

$$
[V, \lambda, p]_{X}\left(\Delta^{m}, E, u\right) \subset[V, \lambda, F, p]_{X}\left(\Delta^{m}, E, u\right) .
$$

\section{References}

[1] Bilgen, T., On statistical convergence, An. Univ. Timisoara Ser. Math. Inform. 32, pp. 3-7, (1994).

[2] Et, M. and Colak, R., On some generalized difference sequence spaces , Soochow J. Math., 21, pp. 377-386, (1965).

[3] Et, M., Altin, Y. and Altinok, H., On some generalized difference sequence spaces defined by a modulus functions , Filomat 17, 23-33, (2003).

[4] Kizmaz, H., On certain sequence spaces, Cand. Math. Bull., 24, pp. 169-176, (1981).

[5] Lindler, L., Uber de la Valle-Pousinsche Summierbarkeit Allgemeiner Orthogonal-reihen, Acta Math. Acad. Sci. Hungar, 16, pp. 375-387, (1965). 
[6] Maddox, I. J., Elements of functional Analysis, Cambridge Univ. Press, (1970).

[7] Malkowsky, E. and Savas, E., Some $\lambda$-sequence spaces defined by a modulus, Archivum Mathematicum, 36, pp. 219-228, (2000).

[8] Savas, E., On some generalized sequence spaces defined by a modulus, Indian J. pure and Appl. Math., 30, pp. 459-464, (1999).

[9] Wilansky, A., Summability through Functional Analysis, North- Holland Math. stnd. (1984).

\section{Kuldip Raj}

School of Mathematics

Shri Mata Vaishno Devi University

Katra-182320, J\&K

India

e-mail : kuldeepraj68@rediffmail.com

and

\section{Sunil K. Sharma}

School of Mathematics

Shri Mata Vaishno Devi University

Katra-182320, J\&K

India

e-mail : sunilksharma42@yahoo.co.in 